\title{
Research on Anomie Behavior of Drivers on Non-physical Isolated Urban Road Based on Game Theory
}

\author{
Meiye $\mathrm{Li}^{1}$, Meiying $\mathrm{Li}^{2}$, Xiaoxia $\mathrm{Hu}^{1}$ and $\mathrm{Ge} \mathrm{Li}^{1}$ \\ ${ }^{1}$ School of Highway, Chang'an University, Middle of South 2rd Ring Road, Xi'an, Shaan'xi Province, China. \\ ${ }^{2}$ School of Control Science and Engineering, Shandong Univiversity, Shandong, China.
}

\begin{abstract}
In order to overtake motor vehicles ahead, some motor vehicles tend to enter non-motorized lane illegally on non-physical isolated urban road, which cause a negative influence on non-motor vehicles. In this paper, this anomie behavior is studied by headway distribution function, fluid dynamics model and game theory. Utilize headway distribution function to calculate the probability of critical gap required for motor vehicles entering nonmotorized lanes, and fluid mechanics model to analysis delay. After that, considering critical gap, delay, management expense and fine for anomie behavior, the time value functions of drivers and cyclists are established and regarded as the payment functions in mixed strategy Nash equilibrium. Finally, according to the result of Nash equilibrium, two optimal probability models are put forward. Under different circumstances of non-motor vehicle flow, motor vehicle speed, management expense and fine for anomie behavior, the first model quantified the optimal probability that drivers commit anomie behavior and the second model quantified the optimal probability that traffic department implements management. Furthermore, models can provide references for the management of anomie behavior on non-physical isolated urban road.
\end{abstract}

\section{Introduction}

Traffic anomie behavior on non-physical isolated urban road refers to the behavior that travelers drive into the non-motorized lane illegally for the fake of overtaking the slow-speed vehicle ahead, which affects traffic safety and efficiency adversely. In China, mixed traffic is a typical characteristic on urban roads, especially nonphysical isolated urban roads. However, due to the lack of traffic administration and existing management methods are unreasonable, the traffic anomie behaviors are very common on urban roads, causing a serious impact on the traffic safety and efficiency.

In the aspect of traffic anomie behavior. In the aspect of anomie behavior. The present study focuses on the misbehavior detection, analysing the influencing factors of the traffic anomie behavior. In term of the misbehavior detection, the data, which was collected through simulating a vehicle crash in different traffic scenarios, was used to design a misbehaviour detection scheme considering two inputs of emergency electronic brake light and lane change ${ }^{[1]}$. Considering the development of connected car, Kamel J. et al. put forward a state-of-theart of misbehavior detection method based on the theory of exchanging Vehicle-to-X communication (V2X) messages ${ }^{[2]}$. In term of the factor analysis, factors of the traffic anomie behavior, such as age, gender, travel purpose, education background and vehicle type, were analysed to built the regression model of traffic anomie behavior $^{[3]}$. Based on the theory of planned behavior, combined with external stimulus, traffic environment and cost pressures a theoretical model of urban traffic anomie behavior on the basis of the theory of planned behavior was constructed ${ }^{[4]}$. According to the difference of time, place and character, the urban traffic anomie behavior is classified and a logistic regression model was established based on traffic participant's physiology, traffic psychology characteristics and traffic environment characteristics $^{[5]}$.

In the aspect of mixed traffic on road section. The present study mainly focuses on simulating the behavior of drivers and analysing the traffic characteristics under different mixed traffic conditions. In term of longitudinal movements of vehicles, several studies has been carried out to evaluate the different car following models under mixed traffic conditions and compare the performance of these car following models ${ }^{[6,7]}$. In term of lateral movements of vehicles, based on the cellular automata theory, some studies has put forward different lane changing models to simulate lane changing behavior under mixed traffic conditions. Considering different types of vehicles take different lane changing rules, a multi-speed lane traffic flow model, which includes motorized vehicle and electric bicycle, was established based on mixed traffic flow of existing NaSch research ${ }^{[8]}$. Due to the car and the truck have different limited deceleration capabilities, Li X. et al. put forward a single lane safety driving (SD) model with limited deceleration 
capability to two-lane SD model, in which car-truck heterogeneous traffic is considered ${ }^{[9]}$. In addition, the traffic characteristics, such as delay, headway, speed, acceleration and deceleration, were analysed under different mixed traffic conditions. Vehicle trajectory data in mixed traffic was extracted from videos, and the mixed traffic flow characteristics, such as speed, acceleration, deceleration and longitudinal spacing, were analysed ${ }^{[10]}$. Traffic volume and speed data was collected for various sections of six lanes and four lane roads, Shah P M. et al. provided an analytical approach to speeds of heterogeneous system of traffic of the various sections ${ }^{[11]}$. Due to the variety of vehicles with their different static and dynamic characteristics. An attempt was made to study the effect of static and dynamic PCU values on the capacity analysis of four lane divided urban roads and to study the effect of proportion of two-wheeler on PCU values $^{[12]}$. Under mixed traffic conditions, the carfollowing models such as Gipps, Intelligent Driver Model (IDM), Krauss Model and Das and Asundi were selected to analyse the capacity, PCU estimation and Level of Service (LOS) ${ }^{[13]}$.

In the aspect of anomie behavior and mixed traffic, present studies mainly focus on the present situation description, reason exploration and influence analysis. The significance of these studies is to improve the traffic condition. However, the measures of controlling the anomie behavior and improving the mixed traffic are insufficient. In order to put forward reasonable suggestions, the analysis of behaviors of traffic participants are needed. In fact, anomie behavior on nonphysical isolated urban road was a two-party game process. In this two-party game process, players are motor vehicles and non motorized vehicles.

In this paper, the anomie behavior, motor vehicles drive into non-motorized lane illegally for the sake of overtaking, was studied by headway distribution function, fluid dynamics model and game theory. Considering critical gap, delay, management expense and fine for anomie behavior, the time value functions of drivers and cyclists were established and regarded as the payment functions in mixed strategy Nash equilibrium. Finally, under different circumstances of motor vehicle speed, management expense, fine for anomie behavior and time value of two players, two optimal probability models were put forward. The first model quantified the optimal probability that drivers committed anomie behavior and the second model quantified the optimal probability that the traffic department implements the management. Moreover, the models could provide references for the management of anomie behavior on non-physical isolated urban road.

\section{Analysis of anomie behavior of motor vehicle}

\subsection{Description of driving process}

In order to overtake motor vehicles ahead, some motor vehicles tend to enter non-motorized lane illegally on non-physical isolated urban road. This process is showed in Figure 1 and described as follows: (1) The road form is a single lane, and there is no physical isolation facilities between the motor lane and the non motorized lane; (2) Distribution of non-motor vehicle is discrete and there is some low-speed motor vehicles on the motor lane. In order to overtake the low-speed motor vehicles ahead, rear motor vehicle waits for the suitable non-motor vehicle spacing to enter the non-motorized lane. If the distance between group $k$ non motorized vehicles and group $k+1$ non motorized vehicles is suitable, the rear motor vehicle will drive into the non-motorized lane for the sake of overtaking the vehicle ahead and drive back to motor lane. The distance that just meets the need of overtaking is defined as critical gap in this paper; (3) When the rear motor vehicle drove into the nonmotorized lane, considering the driving safety, group $k+1$ non motorized vehicles will brake until the longitudinal distance between motor vehicle and reders does not meet the safety requirements, and the aggregated wave will be formed; (4) After that, group $k+1$ non motorized vehicles will speed up, and dissipation wave will be formed.

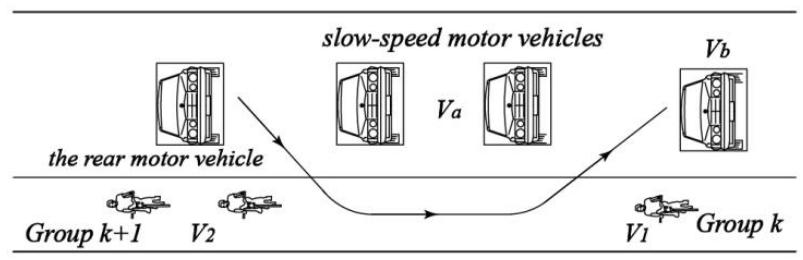

Figure 1. Process of anomie mixed traffic.

In this paper, group $k+1$ non motorized vehicles and the rear motor vehicle are selected as research objective, the change process of traffic flow can be abstracted as: deceleration (aggregated wave) - - following - acceleration (dissipation wave).

In reality, the rear motor vehicle adopts anomie behavior or not is related to the management measures of traffic department. It's easy to know that there is a game between the motor vehicle and non motorized vehicles, so the game model of motor vehicle and non motorized vehicles is needed. There are two players in the game, include motor vehicle and non motorized vehicles. However, considering that slow-moving traffic is advocated by urban policy and protecting the "non-motor vehicle right" is an important principle in traffic management, so traffic management department can represent the interests of non-motor vehicles. Finally, the players in the game are motor vehicle and traffic management department. The purpose of motor vehicles is passing through the road as fast as possible, which means time saving, and the purpose of traffic management department is supervising the anomie behavior of motor vehicle drivers and reducing the delay of non-motor vehicles.

\subsection{Critical gap}

Headway is the time interval between two adjacent vehicles in car-following, which represents the time available to the driver of the following vehicle to reach the same level of deceleration as the lead vehicle in case 
it brakes ${ }^{[14]}$.In the past, the research on the headway was mostly based on the motor vehicle, the research on the headway of non-motor vehicle was inadequately. In this paper, the distribution function of the headway of non motorized vehicle can be obtained by fitting with actual survey data, and the distribution function can be described as equation(1).

$$
P(h<t)=\frac{\int_{0}^{t} f(t) d t}{Q}
$$

where, $P$ is the probability that non-motor vehicle headway $h$ is less than $t, f(t)$ is time headway function of non-motor vehicle, and can be obtained by fitting with actual survey data; $Q$ is the number of non-motor vehicles, which passed the survey line during the investigation period.

Assuming that the time just required for a motor vehicle to overtake lower motor vehicles ahead is $\tau$, and $\tau$ is defined as critical gap. Therefore, $h$ larger than $\tau$ is a necessary condition for drivers to overtake lower motor vehicles ahead by way of entering non-motorized lane illegally. According to the equation(1), the probability of occurrence of critical gap can be calculated as follows:

$$
P_{0}(h \geq \tau)=1-\frac{\int_{0}^{\tau} f(t) d t}{Q}
$$

\subsection{Fluid dynamics model}

Lighthill and Whitham put forward fluid dynamics model by comparing traffic flow to fluid, the model regarded the change of traffic density as the water wave and abstract as a traffic wave. When the traffic conditions change, the changes of traffic density will produce a propagation of traffic wave ${ }^{[15]}$.

In this paper, the delay of group $k+1$ non motorized vehicles was studied when a motor vehicle driver committed anomie behavior. As shown in Figure 1, while the anomie motor vehicle entering the non-motor vehicle lane, assuming that the speed of it is $V_{c}$. After it entering the non-motor vehicle lane, the motor vehicle will overtake the front car and the acceleration is $a$. At the same time, if non-motorized riders recognizing that the longitudinal distance between motor vehicle and reders does not meet the safety requirements, the non-motorized riders of group $k+1$ will brake until the longitudinal distance meets the safe longitudinal distance $L_{0}$, after that the speed of non motorized vehicles will increase and dissipation wave is formed. In addition, due to the braking and acceleration time of non motorized vehicle is quite short, we can be assumed that the speed of nonmotor vehicle reduce from $V_{1}$ to $V_{2}$ immediately after braking, and increase from $V_{2}$ to $V_{1}$ immediately after acceleration. The time tA that non-motor vehicle follows motor vehicle can be calculated by the equation(3).

$$
V_{c} t_{A}+\frac{1}{2} a t_{A}^{2}-V_{2} t_{A}=L_{0}
$$

The distance $x_{A}$ that non-motor vehicle follows motor vehicle can be obtained.

$$
x_{A}=V_{2} t_{A}
$$

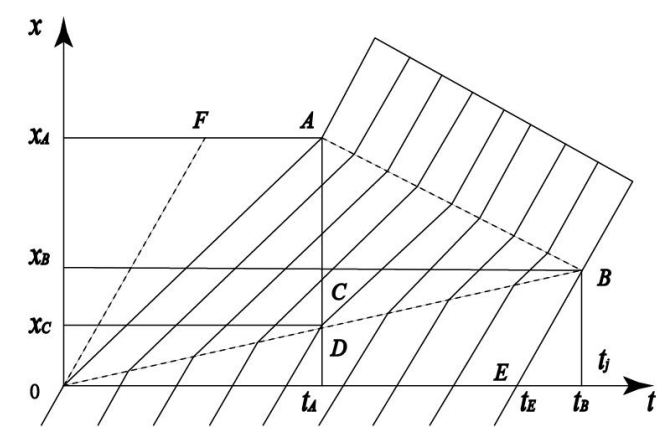

Figure 2. Time and space of motor vehicle.

The time-space trajectory of the group $k+1$ non motorized vehicles is shown in Figure 2. As the speed of non motorized vehicles slow down, the density of group $k+1$ increases from $K_{1}$ to $K_{2}$ and the volume decreases from $Q_{1}$ to $Q_{2}$. At the same time, the aggregated wave is formed and its speed equals to the slope of dashed line $O B$, it can be obtained as follows:

$$
w_{1}=\frac{Q_{2}-Q_{1}}{K_{2}-K_{1}}
$$

When the longitudinal distance between motor vehicle and non motorized vehicles reaches $L_{0}$, non motorized vehicles will speed up, the density of group $k+1$ increases from $K_{2}$ to $K_{3}$ and the volume decreases from $Q_{2}$ to $Q_{3}$. At the same time, the dissipation wave is formed and its speed equals to the slope of dashed line $A B$, it can be obtained as follows:

$$
w_{2}=\frac{Q_{3}-Q_{2}}{K_{3}-K_{2}}
$$

The congestion time is from $t_{0}$ to $t_{A}$. The most congested time is at $t_{A}$ and complete dissipation time is at $t_{B}$, so the difference between $t_{B}$ and $t_{A}$ is the dissipation time $t_{s}$. The congestion duration $t_{j}$ and dissipation time $t_{s}$ can be calculated as follows:

$$
\begin{array}{r}
w_{1}\left(t_{A}+t_{s}\right)=x_{A}+w_{2} t_{s} \\
t_{s}=\frac{x_{A}-w_{1} t_{A}}{w_{1}-w_{2}} \\
t_{j}=t_{A}+t_{s}
\end{array}
$$

The flow of non motorized vehicles, skipped by the aggregated wave in unit time, is $Q_{W l}$. The total number of non motorized vehicles, skipped by the aggregated wave, is $N . Q_{W l}$ and $N$ can be calculated as follows: 


$$
\begin{gathered}
Q_{w 1}=\frac{V_{2}-V_{1}}{\frac{1}{K_{2}}-\frac{1}{K_{1}}} \\
N=Q_{w 1} \cdot t_{j}
\end{gathered}
$$

Finally, the total delay time of group $k+1$ can be calculated by the equation(12).

$$
D=\frac{1}{2} \cdot\left(\frac{x_{A}}{V_{2}}-\frac{x_{A}}{V_{1}}\right) \cdot N
$$

According to the equation(3) to (11), the equation(12) can be converted as follows :

$$
\begin{gathered}
D=\frac{1}{2} \cdot \frac{V_{2}-\frac{Q_{3}-Q_{2}}{K_{3}-K_{2}}}{\frac{Q_{2}-Q_{1}}{K_{2}-K_{1}}-\frac{Q_{3}-Q_{2}}{K_{3}-K_{2}}} \cdot\left(\frac{V_{2}-V_{c}+\sqrt{\left(V_{2}-V_{c}\right)^{2}+2 a L_{0}}}{a}\right)^{2} . \\
\left(1-\frac{V_{2}}{V_{1}}\right) \cdot \frac{V_{2}-V_{1}}{\frac{1}{K_{2}}-\frac{1}{K_{1}}}
\end{gathered}
$$

\section{Game model}

\subsection{Model hypothesis}

Assuming that motor vehicle drivers know that the anomie behavior will be fined, and the penalty for each anomie vehicle is $b$. The probability of traffic management is $\alpha$ and the average cost of supervision and management of each vehicle is $a$. Anomie behavior is bound to be fined once traffic management was implemented. The probability that motor vehicle drivers doesn't commit anomie behavior is $\beta$. In order to simplify the influence of anomie behavior on the payment function of non motor vehicles, the anomie behavior of motor vehicle drivers would be stopped immediately when it was found and fined, so the anomie behavior wouldn't cause a interference to non motor vehicles. If the driver follow the car ahead, the speed of motor vehicle is equal to the vehicle ahead and the speed is denoted as $V_{a}$. To the contrary, if the driver take the strategy to commit anomie behavior, the motor vehicle driver can adopt expected speed and it is denoted as $V_{b}$.

\subsection{Description of players in game}

There are two players in the game, motor vehicle and non-motor vehicle. Considering that slow-moving traffic is advocated by urban policy and protecting the "nonmotor vehicle right" is an important principle in traffic management, so traffic management department can represent the interests of non-motor vehicles.

The purpose of motor vehicles is passing through the road as fast as possible. And the purpose of traffic management department is supervising the anomie behavior of drivers and reducing the delay of non-motor vehicles. Their strategy sets are showed as follows:

(1) Strategy set of traffic management department:

$$
G=\{G 1, G 2\}=\{\text { management, no management }\}
$$

(2) Strategy set of motor vehicle:

$$
H=\{H 1, H 2\}=\{\text { anomie }, \text { no anomie }\}
$$

\subsection{Game strategy analysis}

There are two types of payment functions in the game, including time cost and economic cost. Time cost refer to the time of travel and economic cost contains the cost of management and fine. In order to construct the payment function of each player in the game reasonably, the time cost and economic cost are unified quantification into economic costs by average time value. The game matrix is shown in Table 1.

Table 1. Game matrix of motor vehicle and traffic management department.

\begin{tabular}{|c|c|c|}
\hline $\begin{array}{c}\text { traffic management } \\
\text { department }\end{array}$ & \multicolumn{2}{|c|}{ motor vehicle } \\
\cline { 2 - 3 } management & no anomie & anomie \\
\hline no management & $\left(\mathrm{C}_{1}, \mathrm{C}_{2}\right)$ & $\left(\mathrm{C}_{3}, \mathrm{C}_{4}\right)$ \\
\hline
\end{tabular}

When the traffic management department implements the management and the motor vehicle doesn't adopt anomie behavior. The payment function of traffic management department only includes management fees.

$$
C_{1}=a
$$

The payment function of the motor vehicle is shown as follows.

$$
C_{2}=\frac{L}{V_{a}} V O T_{1} \cdot K
$$

where $L$ indicates the distance between the end of the road and the site where the rear vehicle meets the front motor vehicle with low speed, or the following distance when the rear motor vehicle driver doesn't adopt anomie behavior. $V_{a}$ indicates following speed and it equals to the speed of front vehicle. $V O T_{1}$ is the average time value of motor vehicle driver. $K$ is the average number of passengers on a motor vehicle in the survey area.

When the government implements the management and the motor vehicle commits anomie behavior. The motor vehicle driver would commit anomie behavior once the critical gap occurred. The payment function of traffic management department includes the cost of supervision and management of each vehicle and fine for anomie behavior when the critical gap occurred.

$$
C_{3}=a-P_{0} b
$$

If the critical gap occurred, the payment function of motor vehicle includes the fine when the motor vehicle 
planed to commit anomie behavior but was stopped and the time cost that the motor vehicle following the vehicle ahead and driving through the road after it was fined. If the critical gap didn't occur, the payment function of motor vehicle equals to the time cost that the motor vehicle following the vehicle ahead and driving through the road. So the total payment function of motor vehicle can be described as:

$$
\begin{aligned}
C_{4}=P_{0} & \frac{L}{V_{a}} V O T_{1} \cdot K+P_{0} b+\left(1-P_{0}\right) \frac{L}{V_{a}} V O T_{1} \cdot K \\
= & \frac{L}{V_{a}} V O T_{1} \cdot K+P_{0} b
\end{aligned}
$$

When the government doesn't implement the management and the motor vehicle doesn't adopt anomie behavior. In this condition, non-motor vehicles can pass through the road at the expected speed and no regulatory fees are required. There is no payment for traffic management department.

$$
C_{5}=0
$$

The payment function of motor vehicle equals to the time cost that the rear motor vehicle following the vehicle ahead and driving through the road.

$$
C_{6}=\frac{L}{V_{a}} V O T_{1} \cdot K
$$

When the government doesn't implement the management and the motor vehicle commits anomie behavior. In this condition, the non-motor vehicles is influenced by the anomic motor vehicle. The payment function of traffic management department can be represented by the delay of non-motor vehicles.

$$
C_{7}=D \cdot V O T_{2}
$$

where $\mathrm{VOT}_{2}$ is average time value of non-motor vehicle driver.

Due to the traffic management department doesn't implement the management, there is no fine for anomie behavior. The payment function of motor vehicle equals to the time cost that the rear motor vehicle following the vehicle ahead and driving through the road.

$$
C_{8}=\frac{L}{V_{b}} \cdot V O T_{1} \cdot K
$$

Finally, the payoff matrix of the game can be summarized in the table 2.

Table 2. Payoff matrix of the game.

\begin{tabular}{|c|c|c|}
\hline \multirow{2}{*}{$\begin{array}{c}\text { traffic } \\
\text { management } \\
\text { department }\end{array}$} & \multicolumn{2}{|c|}{ motor vehicle } \\
\cline { 2 - 3 } & no anomie & anomie \\
\hline \multirow{2}{*}{ management } & $\left.\frac{L}{V_{a}} V O T_{1} \cdot K\right)$ & $\left.\frac{L}{V_{a}} V O T_{1} \cdot K+P_{0} b\right)$ \\
\hline no & $(0$, & $\left(D \cdot V O T_{2}, \frac{L}{V} \cdot V O T_{1} \cdot K\right)$ \\
\hline
\end{tabular}

$$
\begin{array}{l|l}
\text { management } & \left.\frac{L}{V_{a}} V O T_{1} \cdot K\right)
\end{array}
$$

\section{Result}

Given the probability $\beta$ that motor vehicle driver doesn't adopt anomie behavior, if the expected utilities of the traffic management department are equal whether it implements the management or not, the following equation can be obtained.

$$
\beta a+(1-\beta) \cdot\left(a-P_{0} b\right)=\beta \cdot 0+(1-\beta) D \cdot V O T_{2}
$$

According to the equation(22), the optimal probability $\beta^{*}$ that motor vehicle driver doesn't adopt anomie behavior can be obtained.

$$
\beta^{*}=1-\frac{a}{P_{0} \cdot b+D \cdot V O T_{2}}
$$

Given the probability $\alpha$ that the traffic management department implements the management, if the expected utilities of the motor vehicle drivers are equal whether they adopt anomie behavior or not, the following equation can be obtained.

$$
\begin{gathered}
\alpha \frac{L}{V_{a}} V O T_{1} \cdot K+(1-\alpha) \cdot \frac{L}{V_{a}} V O T_{1} \cdot K= \\
\alpha \cdot\left(\frac{L}{V_{a}} V O T_{1} \cdot K+P_{0} b\right)-(1-\alpha) \cdot \frac{L}{V_{b}} V O T_{1} \cdot K
\end{gathered}
$$

According to the equation(24), the optimal probability $\alpha^{*}$ that the traffic management department implements the management can be obtained.

$$
\alpha^{*}=\frac{1}{\frac{P_{0} b}{\left(\frac{1}{V_{a}}-\frac{1}{V_{b}}\right) \cdot L \cdot V O T_{1} \cdot K}+1}
$$

The solution of the mixed strategy Nash equilibrium is $\left(\alpha^{*}=\frac{1}{\frac{P_{0} b}{\left(\frac{1}{V_{a}}-\frac{1}{V_{b}}\right) \cdot L \cdot V O T_{1} \cdot K}+1}, \beta^{*}=1-\frac{a}{P_{0} \cdot b+D \cdot V O T_{2}}\right)$, where $P_{0}(h \geq \tau)=1-\frac{\int_{0}^{\tau} f(t) d t}{Q}, D=\frac{1}{2} \cdot \frac{V_{2}-\frac{Q_{3}-Q_{2}}{K_{3}-K_{2}}}{\frac{Q_{2}-Q_{1}}{K_{2}-K_{1}}-\frac{Q_{3}-Q_{2}}{K_{3}-K_{2}}}$. $\left(\frac{V_{2}-V_{c}+\sqrt{\left(V_{2}-V_{c}\right)^{2}+2 a L_{0}}}{a}\right)^{2} \cdot\left(1-\frac{V_{2}}{V_{1}}\right) \cdot \frac{V_{2}-V_{1}}{\frac{1}{K_{2}}-\frac{1}{K_{1}}}$.

\section{Conclusion}




\subsection{Optimal probability model of the Traffic department}

On the non-physical isolated urban road, the optimal $\operatorname{probability}\left(\alpha^{*}\right)$ that traffic department implements the management is:

$$
\alpha^{*}=\frac{1}{\frac{\left(1-\frac{\int_{0}^{\tau} f(t) d t}{Q}\right) b}{\left(\frac{1}{V_{a}}-\frac{1}{V_{b}}\right) \cdot L \cdot V O T_{1} \cdot K}+1}
$$

Under different circumstances of time value of motor vehicle driver, motor vehicle speed, management expense, fine for anomie behavior, non-motor vehicle flow and the passenger number of motor vehicles, the equation(26) quantified the optimal probability that the traffic management department implements management.

For the traffic management department. On one hand, with the increase of average time value of motor vehicle drivers $\left(V O T_{1}\right)$ and average passenger number of motor vehicles $(K)$, the optimal probability $\left(\alpha^{*}\right)$ that the traffic management department implements management is increasing, which means $\alpha^{*}$ is closely related to the development of regional economy and the travel habits of residents. One the another hand, with the increase of the fine for anomie behavior $(b)$, the the optimal probability $\left(\alpha^{*}\right)$ that the traffic management department implements management is decreasing, which means motor vehicle drivers tend to comply with the traffic rules if the fine is big enough.

\subsection{Optimal probability model of the motor vehicle}

On the non-physical isolated urban road, the optimal $\operatorname{probability}\left(\beta^{*}\right)$ that the rear motor vehicle driver doesn't adopt anomie behavior is:

$$
\begin{gathered}
\beta^{*}=1-a /\left[\left(1-\frac{\int_{0}^{\tau} f(t) d t}{Q}\right) \cdot b+\frac{1}{2} \cdot \frac{V_{2}-\frac{Q_{3}-Q_{2}}{K_{3}-K_{2}}}{\frac{Q_{2}-Q_{1}}{K_{2}-K_{1}}-\frac{Q_{3}-Q_{2}}{K_{3}-K_{2}}} .\right. \\
\left.\left(\frac{V_{2}-V_{c}+\sqrt{\left(V_{2}-V_{c}\right)^{2}+2 a L_{0}}}{a}\right)^{2} \cdot\left(1-\frac{V_{2}}{V_{1}}\right) \frac{V_{2}-V_{1}}{\frac{1}{K_{2}}-\frac{1}{K_{1}}} V_{1}\right]
\end{gathered}
$$

Under different circumstances of law enforcement cost of each motor vehicle, average time value of nonmotor vehicle riders, motor vehicle speed, management expense, fine for anomie behavior, non-motor vehicle flow and the average passenger number of motor vehicles, the equation(27) quantified the optimal probability that motor vehicle driver doesn't adopt anomie behavior.
For the motor vehicle driver. On one hand, with the increase of law enforcement cost of each motor vehicle $(a)$, the optimal probability that motor vehicle driver doesn't adopt anomie behavior is decreasing, which means motor vehicle drivers are inclined to commit anomie behavior and drive into the nonmotorized lane illegally for the fake of overtaking the low-speed vehicle ahead when the law enforcement cost was at a low level. One the another hand, with the increase of the fine for anomie behavior $(b)$, the optimal probability that motor vehicle driver doesn't adopt anomie behavior is increasing.

\section{References}

1. A. A. Ardo, A. Zainal, F. A. Ghaleb. Indian J. Sci. Technol. 9 (2016)

2. J. Kamel, A. Kaiser, I. Jemaa. VTC2018-Spring. (2018)

3. R. W. van der Heijden, S. Dietzel, T. Leinmüller. arXiv preprint arXiv. (2016)

4. Li Gaofeng. CNKI. (2016)

5. Yi Zige. CNKI. (2016)

6. C.H. Naveen Kumar. M. Tech Project Report (2013)

7. V. Kanagaraj, G. Asaithambi, C.H.N. Kumar. Procedia-Social and Behavioral Sciences. 104 (2013)

8. You Shiguang, Xiao Wen, Chen Yuguang. Highway Engineering. 3 (2017)

9. Li Xin, Li Xingang, Xiao Yao, Jia Bin. Phys. A. 451 (2016)

10. V. Kanagaraj, G. Asaithambi, T. Toledo. Transp. Res. Rec. NO.2491 (2015)

11. P.M. Shah, N. Gupta. Indian J. Sci. Technol. 9 (2016)

12. C. Chauhan, S. Gupte, K. Bhatt. IJSRSET. 3 (2017)

13. G. Asaithambi, V. Kanagaraj, K.K. Srinivasan. Transportation letters. 10 (2018)

14. Xu Jiqian, Chen Xuewu. Fundamental Traffic Engineering (Highway Traffic Press. 2008)

15. Chen Kuanmin, Yan Baojie. Highway Capacity Analysis (China Communications Press, 2011) 